
A. ZÜTTEL
P. SUDAN
P. MAURON
P. WENGER

\section{Model for the hydrogen adsorption on carbon nanostructures}

\author{
University of Fribourg, Physics Department, Pérolles, 1700 Fribourg, Switzerland
}

\section{Received: 9 September 2003/Accepted: 30 September 2003 Published online: 9 March 2004 • (C) Springer-Verlag 2004}

ABSTRACT The hydrogen sorption capacity of carbon nanostructures was for several years a very controversial subject. Theoretical models have been published demonstrating a great potential for a large hydrogen sorption capacity of carbon nanostructures. Here we present a simple empirical model where condensation of hydrogen as a monolayer at the surface of nanotubes as well as bulk condensation in the cavity of the tube is assumed. The maximum potential amount of hydrogen absorbed according to the model was calculated to be $2.28 \times 10^{-3}$ mass $\% S\left[\mathrm{~m}^{2} \mathrm{~g}^{-1}\right]=3.0$ mass $\%$ for the adsorption of a monolayer hydrogen at the surface. The condensation of hydrogen in the cavity of the tube leads to a potential absorption for single wall nanotubes starting at 1.5 mass $\%$ and increasing with the diameter of the tubes. The experimentally measured hydrogen capacity of the nanotube samples correlates with the B.E.T. specific surface area. The slope of the linear relationship is $1.5 \times 10^{-3}$ mass $\% / \mathrm{m}^{2} \mathrm{~g}^{-1}$. Therefore, the extrapolated maximum discharge capacity of a carbon sample is 2 mass $\%$. Furthermore, it can be concluded, that the hydrogen sorption mechanism is related to the surface of the sample, i.e. a surface adsorption process.

PACS 81.05.Uw; 81.07.De; 82.33.Pt

\section{Carbon nanotubes}

In 1991 Iijima [1] described for the first time the new form of carbon called carbon nanotubes (CNT). Carbon nanotubes are formed by rolled graphite sheets, with an inner diameter starting from $0.7 \mathrm{~nm}$ up to several $\mathrm{nm}$ and a length of ten to one hundred $\mu \mathrm{m}$. The CNTs are described as usually closed on both sides by a hemisphere, i.e. half of a fullerene. Tubes formed by only one single graphite layer are called single wall nanotubes (SWNT). Tubes consisting of multiple concentric graphite layers are called multi-wall nanotubes (MWNT). The interlayer distance in MWNTs is close to the interlayer distance in graphite, which is equal to half of the unit cell parameter $c(0.5 c=0.3355 \mathrm{~nm})$. The diameter of SWNTs varies from 0.671 to $3 \mathrm{~nm}$, whereas MWNTs show typical diameters of 30 to $50 \mathrm{~nm}$. The helicity of the nanotubes is usually described by the Hamada vector [2], which

Fax:+41-26/300-9747, E-mail: andreas.zuettel@unifr.ch indicates how the graphene sheet is rolled up along a lattice vector with components $(n, m)$. The values of the integers $n$ and $m$ identify the general geometry of SWNT. The tubes with $n=m$ are named "armchair"; tubes with either $n=0$ or $m=0$ are named "zigzag"; all others have chiral symmetry [3]. SWNT tend to agglomerate and form bundles of several ten nanotubes. The nanotubes in the bundles are in 2-dimensions closed packed and the intertube distance is $0.334 \mathrm{~nm}$ [4]. Carbon fibers and carbon nanotubes are similar materials except for their inner structure. Carbon fibers consist of graphite platelets stacked together with an interlayer spacing of $0.3355 \mathrm{~nm}$ in various orientations with respect to the fiber axis, giving rise to assorted conformations. Three types of carbon fibers can be distinguished. In the platelet like carbon fiber the area vector of the graphene sheets is parallel to the axis of the fiber. This type of fiber can easily be distinguished from nanotubes since they do not exhibit a concentric hole, i.e. are not tube like and the shadow lines in the TEM images are perpendicular to the fiber axis. The ribbon like fibers consist of continuous graphene layers along the axis of the fiber. This type of fiber exhibits a very similar pattern in the TEM image just like the bundles of single wall nanotubes. The herringbone fibers are an intermediate type of the above described fibers and consist of graphite platelets with an area vector oriented $45^{\circ}$ to the axis of the fiber.

In this paper, we discuss the hydrogen absorption and desorption from nanostructured carbon and nanotube samples. First a model based on a hypothetical condensation mechanism is presented and the possible amount of absorbed hydrogen is calculated. This is followed by a chapter on the gas phase hydrogen absorption and electrochemical hydrogen absorption.

\section{$2 \quad$ Hydrogen adsorption on graphite}

Graphite has a hexagonal structure with an in-plane lattice parameter $a=0.24612 \mathrm{~nm}$ and an interplanar lattice parameter $c=0.6709 \mathrm{~nm}$, therefore the interplanar separation is $0.5 c=0.3355 \mathrm{~nm}$ and the $\mathrm{C}-\mathrm{C}$ bond length is $a / \sqrt{3}=$ $0.14210 \mathrm{~nm}$ at $298.15 \mathrm{~K}$. Using the density of graphite $\varrho=$ $2267 \mathrm{~kg} \mathrm{~m}^{-3}$ and assuming that all the atoms are in one single plane the maximum specific surface area is $S_{1}=1315 \mathrm{~m}^{2} \mathrm{~g}^{-1}$ or if both sides of the plane are considered $S_{2}=2630 \mathrm{~m}^{2} \mathrm{~g}^{-1}$. For a nanotube sample, the specific surface area exposed to 


\begin{tabular}{crrrrc}
\hline No. of Shells & $N_{\text {tot }}$ & $N_{\text {surf }}$ & Surf [\%] & $S_{1}\left[\mathrm{~m}^{2} / \mathrm{g}\right]$ & $S_{2}\left[\mathrm{~m}^{2} / \mathrm{g}\right]$ \\
\hline 1 & 20 & 20 & 100.0 & 1315 & 2630 \\
2 & 59 & 40 & 66.7 & 877 & 1315 \\
3 & 119 & 59 & 50.0 & 658 & 877 \\
4 & 198 & 79 & 40.0 & 526 & 658 \\
5 & 297 & 99 & 33.3 & 438 & 526 \\
6 & 415 & 119 & 28.6 & 376 & 438 \\
7 & 554 & 138 & 25.0 & 329 & 376 \\
8 & 712 & 158 & 22.2 & 292 & 329 \\
9 & 890 & 178 & 20.0 & 263 & 292 \\
10 & 1088 & 198 & 18.2 & 239 & 263 \\
\hline
\end{tabular}

TABLE 1 Properties of nanotubes as a function of the number of shells: The total number of atoms $N_{\text {tot }}$, the corresponding number of surface atoms $N_{\text {surf }}$, the surface atoms in [\%], the outer $\left(S_{1}\right)$ and the total $\left(S_{2}\right)$ specific surface area

the gas depends on the number of shells. Table 1 shows the specific outer surface area and the total specific surface area, i.e. the outer and the inner surface of the tube, of nanotubes as a function of the number of shells.

The adsorption of a gas on a surface is a consequence of the field force at the surface of the solid, called the adsorbent, which attracts the molecules of the gas or vapor, called adsorbate. The forces of attraction emanating from a solid may be of two main kinds, physical or chemical, and they give rise to physical, i.e. Van der Waals, or chemisorption, respectively. We will focus on two types of gases adsorbing on carbon materials, hydrogen gas, and nitrogen gas. Nitrogen gas is widely used for the determination of the specific surface area of samples, and is referred to as the Brunauer, Emmett and Teller (BET) [5] method. Due to the dispersion forces [6], the most stable position for an adsorbed molecule is situated with its center at a distance of about 1 molecular diameter from the surface as the attractive field rapidly diminishes at greater distances. Once a monolayer of adsorbate molecules is formed the gaseous molecule interacts with the surface of the liquid or solid adsorbate. Therefore, the binding energy of the second layer of adsorbate molecules is similar to the latent heat of sublimation or vaporization of the adsorbate. Consequently, the adsorption at a temperature equal or greater to the boiling point of the adsorbate at a given pressure leads to the adsorption of one single monolayer.

In order to calculate the quantity of adsorbate in the monolayer we use the density of the liquid adsorbate and compute the volume of the molecule.

$V_{\mathrm{Ad}}=\frac{M_{\mathrm{Ad}}}{\varrho_{\mathrm{Ad}} N_{\mathrm{A}}}$,

where $M_{\mathrm{Ad}}$ stands for the molecular mass of the adsorbate and $\varrho_{\text {Ad }}$ for the density of the liquid adsorbate, $N_{\mathrm{A}}$ for the Avogadro constant $\left(N_{\mathrm{A}}=6.0220 \times 10^{23} \mathrm{~mol}^{-1}\right)$. $V_{\mathrm{Ad}}$ is the volume reserved for each molecule in the liquid. With the assumption that the molecules are spherical and closed-packed the volume of the sphere representing the molecule is by a factor of 0.7405 smaller than $V_{\mathrm{Ad}}$.

$V_{\mathrm{M}}=\frac{\pi}{3 \sqrt{2}} V_{\mathrm{Ad}}=\frac{\pi}{3 \sqrt{2}} \frac{M_{\mathrm{Ad}}}{\varrho_{\mathrm{Ad}} N_{\mathrm{A}}}$

From the volume of the spherical molecule the diameter $d_{\mathrm{M}}$ can be calculated and assuming a closed package of the molecules in a two dimensional layer at the surface, each molecule occupies the surface area $S_{\mathrm{M}}$.

$$
\begin{aligned}
& d_{\mathrm{M}}=\sqrt[3]{\frac{6 V_{\mathrm{M}}}{\pi}}=\sqrt[3]{\frac{\sqrt{2} M_{\mathrm{Ad}}}{\varrho_{\mathrm{Ad}} N_{\mathrm{A}}}} \\
& S_{\mathrm{M}}=\frac{\sqrt{3}}{2}\left(d_{\mathrm{M}}\right)^{2}=\frac{\sqrt{3}}{2}\left(\frac{\sqrt{2} M_{\mathrm{Ad}}}{\varrho_{\mathrm{Ad}} N_{\mathrm{A}}}\right)^{\frac{2}{3}}
\end{aligned}
$$

The above parameters for hydrogen and nitrogen are summarized in Table 2. The condensation of a monolayer of hydrogen on a graphene sheet with a specific surface area of $S_{1}=1315 \mathrm{~m}^{2} \mathrm{~g}^{-1}$ leads to

$\frac{m\left(\mathrm{H}_{2}\right)}{m(\mathrm{C})}=\frac{S_{1} M_{\mathrm{Ad}}}{S_{\mathrm{M}} N_{\mathrm{A}}}$

$\left.m\left(\mathrm{H}_{2}\right) / m(\mathrm{C})\right)=0.03085$. This converts according to

$c[\operatorname{mass} \%]=\frac{1}{1+\frac{m(\mathrm{C})}{m\left(\mathrm{H}_{2}\right)}}$

to a maximum concentration of $2.28 \times 10^{-3}$ mass $\% S\left[\mathrm{~m}^{2} \mathrm{~g}^{-1}\right]$ $=3.0$ mass $\%(\mathrm{H} / \mathrm{C}=0.18)$ hydrogen on carbon.

The main difference between carbon nanotubes and high surface area graphite is the curvature of the graphene sheets and the cavity inside the tube. The surface area for the adsorption of gas molecules on the outer side of a nanotube is larger as compared to the surface area available on a flat graphene sheet. This is because the centers of the hydrogen molecules are located at a distance $d$ from the nanotube which is greater than the diameter of the nanotube due the covalent diameter of the carbon atoms and the hydrogen molecules. However, the curvature of the graphene sheet in nanotubes leads to a weaker adsorption potential, due to the larger distance to the second next neighbor carbon atoms.

\section{$3 \quad$ Adsorption in micropores}

In microporous solids with capillaries which have a width not exceeding a few molecular diameters, the potential fields from opposite walls will overlap so that the attractive force acting on adsorbate molecules will be increased as compared with that on an open surface. Stan and Cole [7] used the Feynman (semiclassical) effective potential approximation to calculate the adsorption potential and the amount of hydrogen adsorbed on a zigzag nanotube $(13,0)$ with a diameter of $1.018 \mathrm{~nm}$. The adsorption potential was found to be $9 \mathrm{~kJ} \mathrm{~mol}^{-1}$ for hydrogen molecules inside the nanotubes at $50 \mathrm{~K}$, the potential is about $25 \%$ higher as compared to the flat surface of graphite due to the curvature of the surface and therefore an increased number of carbon atoms interacting with the hydrogen molecule. The ratio of hydrogen adsorbed in the tube to that on a flat surface decreases strongly with increasing temperature and is 10000 at $50 \mathrm{~K}$ and 100 at $77 \mathrm{~K}$ (values taken from figure).

Regardless of the potential inside the tube it is very unlikely that the hydrogen molecules would contract more inside the tube as compared to their liquid state. If we assume a condensation of the hydrogen gas in the cavity of a nanotube, we 
can express the ratio of the mass of hydrogen to the mass of carbon as

$$
\frac{m\left(\mathrm{H}_{2}\right)}{m(\mathrm{C})}=\frac{S_{1} d_{\mathrm{NTT}} \varrho_{\mathrm{Ad}}}{4}
$$

and the ratio for single wall nanotubes with a diameter of $d_{\mathrm{NT}}=0.671 \mathrm{~nm}$ is $m\left(\mathrm{H}_{2}\right) / m(\mathrm{C})=0.0156$ which results in $c=1.54$ mass \% $(\mathrm{H} / \mathrm{C}=0.09)$. However, the available volume inside a nanotube is, due to the space occupied by the carbon atoms with a covalent radius of approximately $0.077 \mathrm{~nm}$ [8], much smaller than the estimated volume in the above model.

The calculated amount of hydrogen absorbed at the surface and in the cavity of the carbon nanotubes are shown in Fig. 1. The surface absorption is highest for the CNT with the largest specific surface area, i.e. the SWNT. The bulk absorption in the cavity is proportional to the diameter of the tubes and is also highest for SWNT. Furthermore, the mass $\%$ of adsorbed hydrogen in the cavity of the tube increases with the tube diameter. This theoretical consideration is based on the assumption that the hydrogen would condense in the nanotube sample. The critical temperature for nitrogen and hydrogen is $126.2 \mathrm{~K}$ and $32.98 \mathrm{~K}$, respectively. Therefore, nitrogen may condense in small pores when the surface area of the material is measured using the B.E.T method $(T=78 \mathrm{~K})$ and the calculated surface area determined from the isotherms may be much larger than the real surface area of the sample. However, hydrogen would only condense below the critical temperature of $33 \mathrm{~K}$.

Rzepka et al. [9] used a grand canonical ensemble Monte Carlo program to calculated the amount of absorbed hydrogen for a slit pore and a tubular geometry. The amount of absorbed hydrogen depends on the surface area of the sample and the maximum is at 0.6 mass $\%(T=300 \mathrm{~K}$, $p=6 \mathrm{MPa})$. The calculation was verified experimentally with an excellent agreement. At a temperature of $77 \mathrm{~K}$ the amount of absorbed hydrogen is about one order of mag-

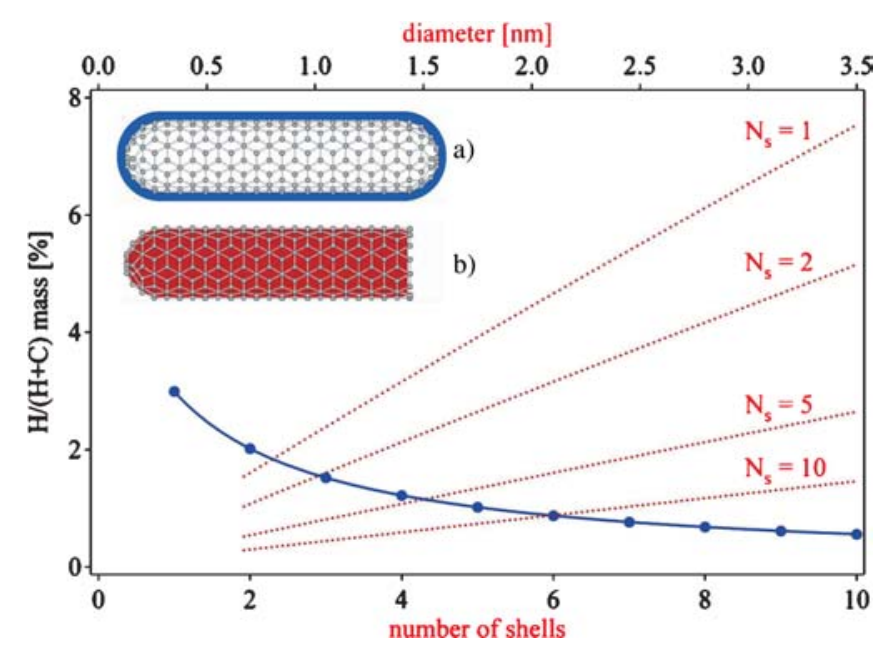

FIGURE 1 Calculated amount of adsorbed hydrogen on carbon nanotubes assuming condensation of hydrogen. a) Monolayer adsorbed at the surface of nanotubes as a function of the number of shells (markers and line, axis: left and bottom), b) Hydrogen condensed in the cavity of nanotubes as a function of the diameter (dashed lines, axis: left and top) for various number of shells, $N_{\mathrm{S}}=1(\mathrm{SWNT}), N_{\mathrm{S}}=2, N_{\mathrm{S}}=5, N_{\mathrm{S}}=10$ (dotted line, axis: left and top) nitude higher as compared to $300 \mathrm{~K}$. Williams and Eklund [10] performed a Monte Carlo simulation of $\mathrm{H}_{2}$ physisorption in finite-diameter carbon SWNT ropes and found an increasing amount of adsorbed hydrogen with decreasing temperature from 1.4 mass $\%(p=10 \mathrm{MPa}, T=300 \mathrm{~K})$ to 9.6 mass $\%$ ( $p=10 \mathrm{MPa}, T=77 \mathrm{~K})$. For lower hydrogen pressure this range is shifted to considerably lower amounts of adsorbed hydrogen, i.e. 0.2 mass $\%(p=1 \mathrm{MPa}, T=300 \mathrm{~K})$ to 5.9 mass $\%(p=10 \mathrm{MPa}, T=77 \mathrm{~K})$. The heat of vaporization of hydrogen is $H_{\text {vap }}=222.5 \mathrm{~J} \mathrm{~mol}^{-1}$ [8] at the boiling point $(T=20.28 \mathrm{~K})$. Using the results in Table 2 , the heat of vaporization can be expressed as $2.85 \mathrm{~mJ} \mathrm{~m}^{-2}$ for the adsorption of a monolayer of hydrogen on a graphene sheet. This value is rather small and leads to a maximum temperature change of $5 \mathrm{~K}$ in the sample in an adiabatic system. Figure 2 shows the volumetric hydrogen density versus the gravimetric hydrogen density in hypothetical SWNT's with various diameters according to the above-described model based on condensed hydrogen in the cavity of the nanotube. According to the criteria described by Switendick [11] the hydrogen atoms in metal hydrides have an interatomic distance of at least $0.21 \mathrm{~nm}$. This corresponds to an atomic volume of $0.00485 \mathrm{~nm}^{3}$ and a maximum volumetric density of $187 \mathrm{~kg} \mathrm{~m}^{-3}$. The volumetric density of hydrogen in hydrocarbons reaches 100 to $110 \mathrm{~kg} \mathrm{~m}^{-3}$. The line for nanotubes is universal; i.e. all types of nanotubes are on this line as long as the hydrogen adsorption occurs due to condensation inside the

\begin{tabular}{lcl}
\hline Property & $\mathrm{N}_{2}$ & $\mathrm{H}_{2}$ \\
\hline$M\left[\mathrm{~g} \mathrm{~mol}^{-1}\right]$ & 28.0140 & 2.0159 \\
$\varrho\left[\mathrm{g} \mathrm{cm}^{-3}\right]$ & 0.8070 & 0.0708 \\
$V_{\mathrm{M}\left[\mathrm{nm}^{3}\right]}$ & 0.0426 & 0.0350 \\
$d[\mathrm{~nm}]$ & 0.4335 & 0.4059 \\
$S_{\mathrm{M}\left[\mathrm{nm}^{2}\right]}$ & 0.1627 & 0.1427 \\
$1 / S\left[\mathrm{~mol} \mathrm{~m}^{-2}\right]$ & $1.02 \times 10^{-5}$ & $1.16 \times 10^{-5}$ \\
$1 / S\left[\mathrm{~g} \mathrm{~m}^{-2}\right]$ & $2.86 \times 10^{-4}$ & $2.35 \times 10^{-5}$ \\
\hline
\end{tabular}

TABLE 2 The properties of hydrogen and nitrogen as adsorbates: $M$ the molecular mass, $\varrho$ the density of the liquid at the boiling point, $V_{\mathrm{M}}$ the volume of the molecule (7), $d$ the diameter of the molecule, $S_{\mathrm{M}}$ the surface area occupied by the molecule, $1 / S$ the amount of adsorbate per surface area unit in a monolayer

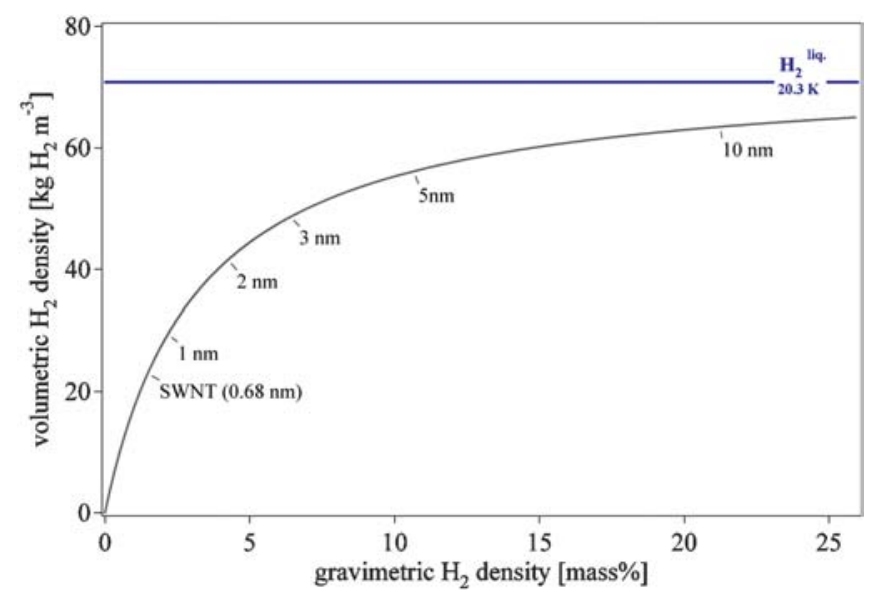

FIGURE 2 Specific volumetric hydrogen density versus the gravimetric hydrogen density for single wall nanotubes with various diameters. The SWNT line represents the calculated values for the cavity filled with liquid hydrogen 
nanotube. Therefore, the only way to increase the volumetric hydrogen density above the density of liquid hydrogen is the dissociation of hydrogen molecules in combination with a tight binding (covalent) or an electron transfer to the host material like in metal hydrides.

\section{4}

\section{Experimental verification}

A large variety of carbon samples were investigated, using a high pressure micro-balance, by Ströbel et al. [12]. The BET $\left(\mathrm{N}_{2}\right)$ surface area of the samples ranges from $100 \mathrm{~m}^{2} \mathrm{~g}^{-1}$ up to $3300 \mathrm{~m}^{2} \mathrm{~g}^{-1}$. The absorbed amount of hydrogen $(p=12.5 \mathrm{MPa}, T=296 \mathrm{~K})$ correlates with the surface area according to the equation $x$ [mass $\%]=$ $5 \times 10^{-4} S\left[\mathrm{~m}^{2} \mathrm{~g}^{-1}\right]$ (taken from Table 1 in [13]) except for the nanofiber samples. The latter exhibit a rather low surface area of approximately $100 \mathrm{~m}^{2} \mathrm{~g}^{-1}$; however, the increase in mass upon hydrogen absorption corresponds to about 1.2 mass \%. The adsorption isotherms measured approximately follow the Langmuir adsorption model. Some isotherms intercept the mass-axis $(p=0)$ at $x=0$, other intercept at a finite mass between 0.2 and 0.4 mass $\%$.

Nijkamp et al. [13] characterised a large number of carbonaceous sorbents using $\mathrm{N}_{2}$ physisorption at $77 \mathrm{~K}$ and up to a pressure of 1 bar. The sorbents were chosen to represent a large variation in surface areas and (micropores) volumes. Both non-porous materials, such as aerosil and graphites, and microporous sorbents, such as activated carbons and zeolites, were selected. The $\mathrm{H}_{2}$-adsorption measurements were performed at $77 \mathrm{~K}$ in the pressure range $0-1$ bar. From adsorption-desorption experiments it is evident that reversible physisorption takes place exclusively with all samples. The amount of adsorbed hydrogen correlates with the specific surface area of the sample (Fig. 3). A few papers on electrochemical measurements at room temperature of hydrogen uptake and release have been published [14-17]. The electrochemical hydrogen absorption is reversible. The maximum discharge capacity measured at $298 \mathrm{~K}$ is 2 mass \% with

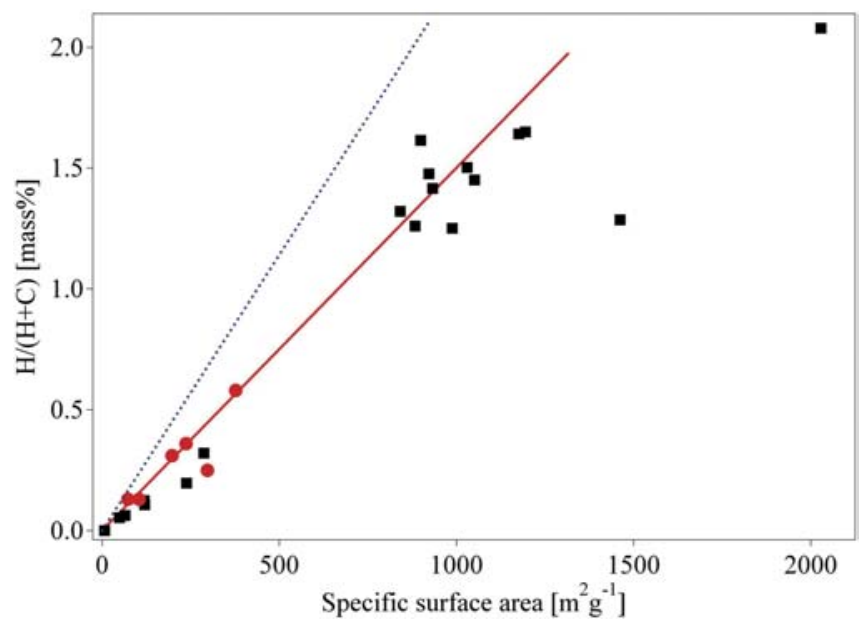

FIGURE 3 Desorbed amount of hydrogen versus the B.E.T. surface area (round markers) of a few carbon nanotube samples including two measurements on high surface area graphite (HSAG) samples [23] together with the fitted line. Data from Nijkamp et al. [13] (rectangular markers) are also shown a very small discharge current (discharge process for $1000 \mathrm{~h}$ ). The few round markers with the fitted line in Fig. 3 are electrochemical results. It is remarkable, that the measurements of the hydrogen uptake in the gas phase at $77 \mathrm{~K}$ exhibit the same quantities as the electrochemical measurements at room temperature $298 \mathrm{~K}$. In the electrochemical charging process hydrogen atoms are left back at the surface of the electrode when the electron transfer from the conductor to the water molecules takes place. The hydrogen atoms recombine to hydrogen molecules. This process goes on until the surface is completely covered with a monolayer of physisorbed $\mathrm{H}_{2}$ molecules. Additional hydrogen does not interact with the attractive Van der Waals forces of the surface. The hydrogen molecules become very mobile and form gas bubbles, which are released from the electrode surface. The formation of a stable monolayer of hydrogen at the electrode surface at room temperature is only possible if either the hydrogen atoms or the hydrogen molecules are immobile, i.e. their surface diffusion has to be kinetically hindered by a large energy barrier probably due to the adsorbed electrolyte $\left(\mathrm{H}_{2} \mathrm{O}\right)$ molecules in the second layer. An other possible reaction path was first reported [18] as a result of density-functional calculation. The result of this calculation is that hydrogen atoms tend to chemisorb at the exterior surface of a nanotube. The atoms can then flip in and recombine to hydrogen molecules finally at high coverage forming a concentric cylinder in the cavity of the nanotube. If the binding energy of the chemisorbed hydrogen is relatively low compared to the energy in hydrocarbons, the absorbed amount of hydrogen is proportional to the surface area of the carbon sample and could also desorb at a rather positive electrochemical potential.

The experimental results are in good agreement with the theoretical estimations if we take into account that the measurements were carried out at a temperature of $77 \mathrm{~K}$ which is still far above the critical temperature of hydrogen of $32 \mathrm{~K}$ and therefore the monolayer of hydrogen is not complete at $77 \mathrm{~K}$. No evidence of an influence of the geometric structure of the nanostructured carbon on the amount of absorbed hydrogen was found. It's quite obvious, that the curvature of nanotubes may only influence the adsorption energy but not the amount of adsorbed hydrogen. Furthermore, all attempts to open the nanotubes and to absorb hydrogen inside the tubes did not show an increased absorption of hydrogen molecules. Theoretical studies beyond the well-known physisorption lead to a large set of various maximum hydrogen absorption capacities. Most of the results were found under special conditions, e.g. at $0 \mathrm{~K}$ or high energy hydrogen atom implantation. No evidence was found for a higher density of hydrogen in and on carbon nanostructures compared to liquid hydrogen at ambient conditions.

Beside the carbon nanostructures, other nanopourus materials have been investigated for hydrogen absorption. The hydrogen absorption of zeolites of different pore architecture and composition, e.g. A, X, Y, was analyzed in the temperature range from $293 \mathrm{~K}$ to $573 \mathrm{~K}$ and pressure range from 2.5 MPa to $10 \mathrm{MPa}$ [19]. Hydrogen was absorbed at the desired temperature and pressure. The sample was then cooled to room temperature and evacuated. Subsequently the hydrogen release upon heating of the sample to the absorption temperature was detected. The absorbed amount of hydrogen 
increased with increasing temperature and increasing absorption pressure. The maximum amount of desorbed hydrogen was found to be 0.08 mass \% for a sample loaded at a temperature of $573 \mathrm{~K}$ and a pressure of $10 \mathrm{MPa}$ [19]. The adsorption behavior indicates that the absorption is due to a chemical reaction rather than physisorption. At liquid nitrogen temperature $(77 \mathrm{~K})$ the zeolites physisorb hydrogen in proportion to the specific surface area of the material. A maximum of 1.8 mass \% of adsorbed hydrogen was found for a zeolite $(\mathrm{NaY})$ with a specific surface area of $725 \mathrm{~m}^{2} \mathrm{~g}^{-1}$ [20]. The low temperature physisorption (type I isotherm) of hydrogen in zeolites is in good agreement with the adsorption model mentioned above for nanostructured carbon. The desorption isotherm followed the same path as the adsorption [20] which indicates that no pore condensation occurred.

5

\section{Hydrogen absorption on carbon}

Nanostructured graphite was investigated by Orimo et al. [21] for hydrogen absorption and desorption. Graphite was ball milled for a maximum of 80 hours in a $1 \mathrm{MPa}$ hydrogen atmosphere. The hydrogen absorbed in the sample was determined by means of oxygen-combustion hydrogen analysis and turned out to be as high as 7.4 mass \%
$(\mathrm{C} / \mathrm{H}=0.95)$. This result shows that high hydrogen absorption can also be realized without the hollow structure of nanotubes. However the hydrogen desorbs only at temperatures above $600 \mathrm{~K} \mathrm{[22]} \mathrm{which} \mathrm{indicates,} \mathrm{that} \mathrm{the} \mathrm{hydrogen} \mathrm{is}$ chemisorbed rather than physisorbed.

The hydrogen absorption properties of the samples in the gas phase were investigated by Züttel et al. [23] by means of a high pressure ( $2 \mathrm{MPa}$ ) absorption and subsequent temperature programmed desorption spectroscopy (TDS). High surface area graphite (HSAG) and SWNT samples exhibit a low temperature hydrogen desorption peak at $105 \mathrm{~K}$ (physisorbed hydrogen) followed by a much smaller peak at $136 \mathrm{~K}$. Above this temperature the hydrogen pressure was continuously decreased showing only a small shoulder at $300 \mathrm{~K}$. The shape of the spectra of the SWNT and the graphite are similar. Above $500 \mathrm{~K}$ the spectra show an increase in hydrogen pressure. We observed, that the increase of the hydrogen pressure in the desorption spectrum becomes more pronounced if the absorption of hydrogen is performed at elevated temperatures $(T>500 \mathrm{~K})$. Therefore, the HSAG sample (high surface area graphite from Timcal) was heated to $600 \mathrm{~K}$ for the hydrogen absorption. Around $100 \mathrm{~K}$ the total pressure is given by the molecular hydrogen partial pressure and hydrogen still contributes significantly to the total pressure up to
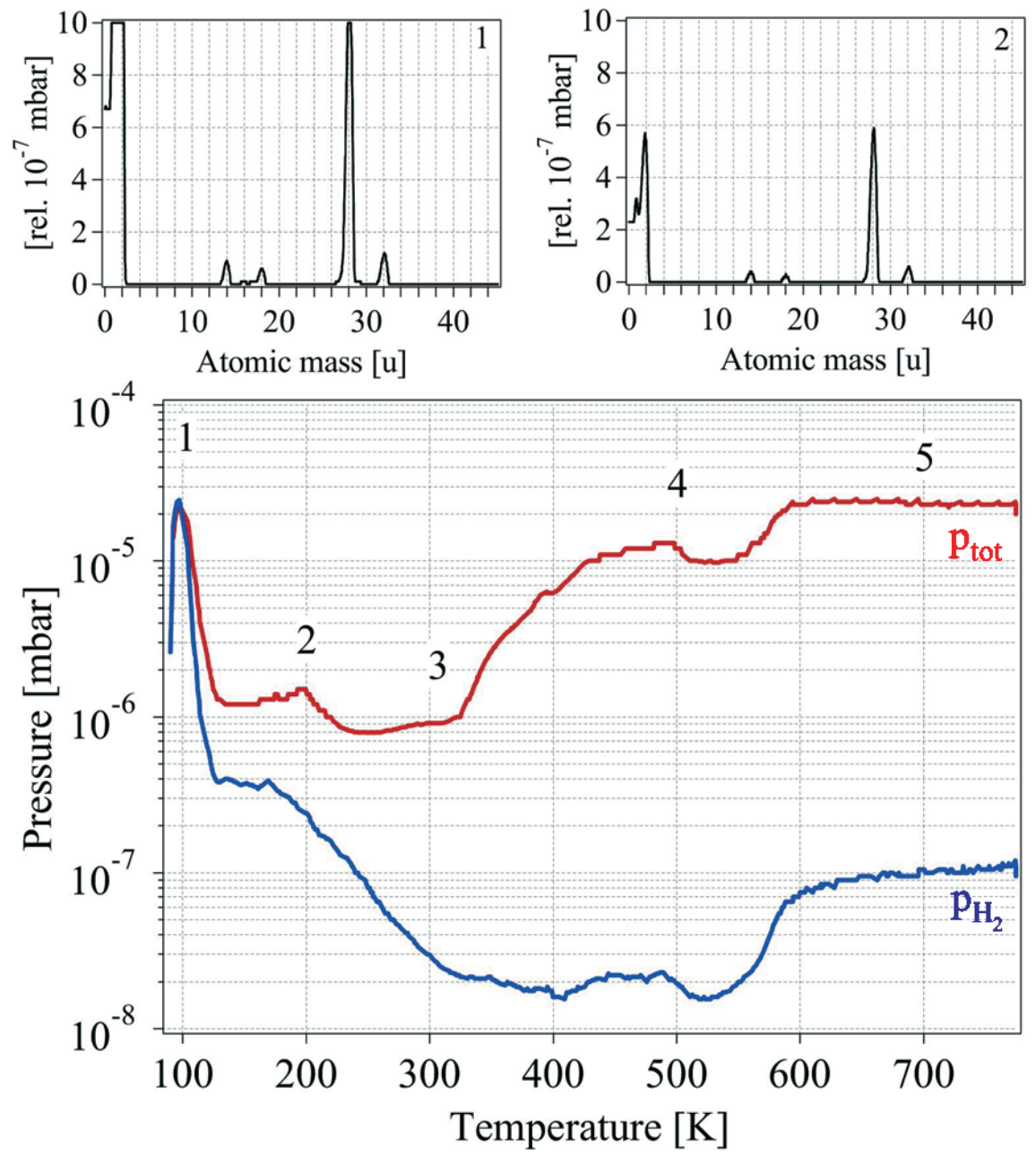
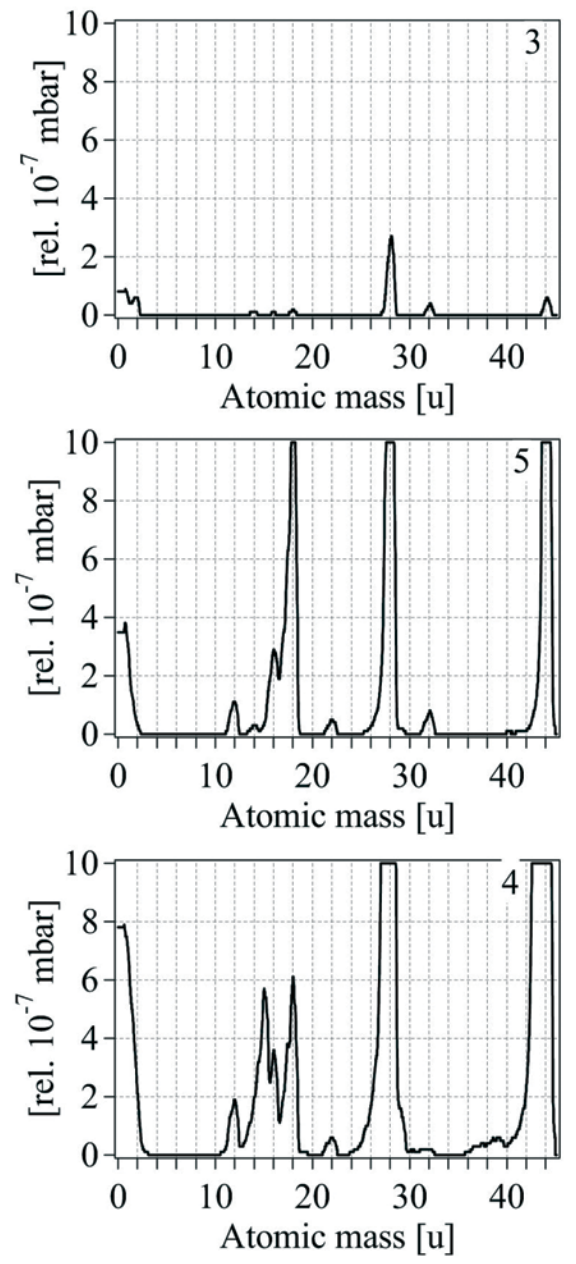

FIGURE 4 Temperature programmed desorption spectra for a high surface area graphite (HSAG 300) sample from Timcal. The sample was heated to 600 K in a hydrogen atmosphere $(2 \mathrm{MPa})$ and subsequently evacuated at $78 \mathrm{~K}$ before the desorption experiment started with a heating rate of $4 \mathrm{~K}$ min ${ }^{-1}$ 
$200 \mathrm{~K}$. At higher temperatures the hydrogen partial pressure is insignificant. The main contribution at temperatures greater than $300 \mathrm{~K}$ to the total pressure are molecules or fragments of molecules with an atomic mass of 12 to $18 \mathrm{u}, 28 \mathrm{u}$ and 42 to $44 \mathrm{u}$. The reason for the occurrence of the large masses are hydrocarbons e.g. $\mathrm{CH}_{4}, \mathrm{C}_{2} \mathrm{H}_{4}, \mathrm{C}_{3} \mathrm{H}_{8}$, which are preferentially formed at higher temperatures and desorb or decompose in the range of $300 \mathrm{~K}$ to $800 \mathrm{~K}$ (Fig. 4). The hydrocarbons are only observed if the absoption of hydrogen is performed at elevated temperatures and not in the case of hydrogen absorption at room temperature. Therefore a considerably high amount of the absorbed hydrogen binds covalently to the carbon sample. Orimo [22] came to the same conclusion for graphite samples which were heavily ball milled in a hydrogen atmosphere.

The interaction of atomic hydrogen and low-energy hydrogen ions with $s p^{2}$-bonded carbon was investigated by Ruffieux et al. [24] on the surfaces of $C_{60}$ multilayer films, single-walled carbon nanotubes, and graphite(0001). These three materials have been chosen to represent $s p^{2}$-bonded carbon networks with different local curvatures and closed surfaces (i.e. no dangling bonds). Chemisorption of hydrogen on these surfaces reduces emission from photoemission features associated with the pi electrons and leads to a lowering of the work function up to $1.3 \mathrm{eV}$. It is found that the energy barrier for hydrogen adsorption decreases with increasing local curvature of the carbon surface. Whereas in the case of $\mathrm{C}_{60}$ and single-walled carbon nanotubes, hydrogen adsorption can be achieved by exposure to atomic hydrogen, the hydrogen adsorption on graphite $(0001)$ requires $\mathrm{H}^{+}$ions of low kinetic energy (similar to $1 \mathrm{eV}$ ). On all three materials, the adsorption energy barrier is found to increase with coverage. Accordingly, hydrogen chemisorption saturates at coverages that depend on the local curvature of the sample and the form of hydrogen (i.e., atomic or ionic) used for the treatment.

\section{6}

\section{Conclusion}

We presented a model where condensation of hydrogen as a monolayer at the surface of nanotubes as well as bulk condensation in the cavity of the tube is assumed. The maximum potential amount of hydrogen absorbed according to the model was calculated to be 3.0 mass \% for the adsorption of a monolayer hydrogen at the surface and therefore proportional to the specific surface area of the carbon sample $2.28 \times 10^{-3}$ mass $\% S\left[\mathrm{~m}^{2} \mathrm{~g}^{-1}\right]$. The condensation of hydrogen in the cavity of the tube leads to a maximum potential absorption for single wall nanotubes starting at 1.5 mass $\%$ and increasing with the diameter of the tubes. Single wall nanotubes with a diameter of $2.2 \mathrm{~nm}$ have the potential to absorb 5 mass $\%$ of hydrogen. The model suffers from the assumption that the hydrogen condenses in the nanotubes although the critical temperature of hydrogen is at $33 \mathrm{~K}$.

The experimentally measured hydrogen capacity of the nanotube samples correlates with the B.E.T. specific surface area. The slope of the linear relationship is $1.5 \times 10^{-3}$ mass $\%$ $S\left[\mathrm{~m}^{2} \mathrm{~g}^{-1}\right]$. Therefore, the extrapolated maximum hydrogen capacity of a carbon sample is 2.0 mass \%. Furthermore, it can be concluded, that the hydrogen sorption mechanism is related to the surface of the sample, i.e. a surface adsorption process.

ACKNOWLEDGEMENTS This work was supported by the Swiss federal office of energy (Bundesamt für Energie, BfE) in contract with the International Energy Agency (IEA), the Swiss Federal Office of Education and Science (BBW), the European Commission (Project FUCHSIA) and the Science Faculty of the University of Fribourg in Switzerland.

\section{REFERENCES}

1 S. Iijima: Nature 354, 56 (1991)

2 N. Hamada, S. Sawada, A. Oshiyama: Phys. Rev. Lett. 68, 54 (1992)

3 M.S. Dresselhaus, G. Dresselhaus, P.C. Eklund: Science of Fullerenes and Carbon Nanotubes (Academic Press, NY 1996)

4 F. Darkrim, D. Levesque: J. Chem. Phys. 109, 4981 (1998)

5 S. Brunauer, P.H. Emmett, E. Teller: J. Amer. Chem. Soc. 60, 309 (1938)

6 F. London: Z. Physik. 63, 245 (1930); Z. Physik. Chem. 11, 222 (1930)

7 G. Stan, M.W. Cole: Low Temp. Phys. 100, 539 (1998)

8 Handbook of Chemistry and Physics, ed. by D.R. Linde, 76th. ed. (CRC Press, 1995-1996)

9 M. Rzepka, P. Lamp, M.A. de la Casa-Lillo: J. Phys. Chem. B102, 10849 (1998)

10 K.A. Williams, P.C. Eklund: Chem. Phys. Lett. 320, 352 (2000)

11 A.C. Switendick: Z. Phys. Chem. N.F. 117, 89 (1979)

12 R. Ströbel, L. Jörissen, T. Schliermann, V. Trapp, W. Schütz, K. Bohmhammel, G. Wolf, J. Garche: J. Power Sources 84, 221 (1999)

13 M.G. Nijkamp, J.E.M.J. Raaymakers, A.J. van Dillen, K.P. de Jong: Appl. Phys. A 72, 619 (2001)

14 C. Nützenadel, A. Züttel, L. Schlapbach: Electronic Properties of Novel Materials, Science and Technology of Molecular Nanostructures, ed. by H. Kuzmany, J. Fink, M. Mehring, S. Roth (Amer. Inst. Phys., NY 1999), p. 462

15 C. Nützenadel, A. Züttel, C. Emmenegger, P. Sudan, L. Schlapbach: Sciences and Applications of Nanotubes, Fundamental Materials Research Series, ed. by M.F. Thorpe (Kluwer Academic Publ./Plenum Press) p. 205

16 A. Züttel, P. Sudan, P. Mauron, C. Emmenegger, T. Kiyobayashi, L. Schlapbach: J. Metastable and Nanocrystalline Materials 11, 95 (2001)

17 S.M. Lee, K.S. Park, Y.C. Choi, Y.S. Park, J.M. Bok, D.J. Bae, K.S. Nahm, Y.G. Choi, S.C. Yu, N. Kim, T. Frauenheim, Y.H. Lee: Synthetic Metals 113, 209 (2000)

18 S.M. Lee, K.H. An, Y.H. Lee, G. Seifert, T. Frauenheim: J. Korean Phys. Soc. 38, 686 (2001); S.M. Lee, Y.H. Lee: Appl. Phys. Lett. 76, 2879 (2000)

19 J. Weitkamp, M. Fritz, S. Ernst: Int. J. Hydrogen Energy 20, 967 (1995)

20 H.W. Langmi, A. Walton, M.M. Al-Mamouri, S.R. Johnson, D. Book, J.D. Speight, P.P. Edwards, I. Gameson, P.A. Anderson, I.R. Harris: J. Alloys Compd. 356-357, 710-715 (2003)

21 S. Orimo, G. Majer, T. Fukunaga, A. Züttel, L. Schlapbach, H. Fujii: Appl. Phys. Lett. 75, 3093 (1999)

22 S. Orimo, T. Matsushima, H. Fujii, T. Fukunaga, G. Majer: J. Appl. Phys. 90, 1545 (2001)

23 A. Züttel, C. Nützenadel, P. Sudan, P. Mauron, C. Emmenegger, S. Rentsch, L. Schlapbach, A. Weidenkaff, T. Kiyobayashi: J. Alloys and Compounds 1, 676 (2002)

24 P. Ruffieux, O. Groning, M. Bielmann, P. Mauron, L. Schlapbach, P. Groning: Phys. Rev. B 66, 245416 (2002) 